\title{
General Relativistic Energy Conditions: The Hubble expansion in the epoch of galaxy formation.
}

\author{
Matt Visser ${ }^{\dagger}$ \\ Physics Department \\ Washington University \\ Saint Louis \\ Missouri 63130-4899 \\ USA
}

26 May 1997; gr-qc/9705070

\begin{abstract}
The energy conditions of Einstein gravity (classical general relativity) are designed to extract as much information as possible from classical general relativity without enforcing a particular equation of state for the stress-energy. This systematic avoidance of the need to specify a particular equation of state is particularly useful in a cosmological setting - since the equation of state for the cosmological fluid in a Friedmann-RobertsonWalker type universe is extremely uncertain. I shall show that the energy conditions provide simple and robust bounds on the behaviour of both the density and look-back time as a function of red-shift. I shall show that current observations suggest that the so-called strong energy condition (SEC) is violated sometime between the epoch of galaxy formation and the present. This implies that no possible combination of "normal" matter is capable of fitting the observational data.
\end{abstract}

PACS: 98.80.-k; 98.80.Bp; 98.80.Hw; 04.90.+e

$\dagger$ E-mail: visser@kiwi.wustl.edu 


\section{Introduction}

The discussion presented in this paper is a model-independent analysis of the misnamed age-of-the-universe problem, this really being an age-of-the-oldeststars problem. In this paper I trade off precision against robustness: I sacrifice the precision that comes from assuming a particular equation of state, for the robustness that arises from model independence. A briefer presentation of these ideas can be found in [1], while a related analysis is presented in [2].

The energy conditions of Einstein gravity (classical general relativity) are designed to side-step, as much as possible, the need to pin down a particular equation of state for the stress-energy. The energy conditions are used, for instance, in deriving many theorems of classical general relativity - such as the singularity theorems of stellar collapse (black hole formation) [3]; the area increase theorem for black holes [3]; the topological censorship theorem [4], and the positive mass theorem [5, 6, 6, 8]. (For a general discussion see, for instance, [3, 9, 10].)

It is well known that these classical energy conditions are violated by small quantum effects, with typical quantum violations being of order $\langle T\rangle_{\text {violation }} \approx$ $\hbar /(G M)^{4}$ 10, 11, 12, 13. These quantum effects are all explicitly of order $\hbar$. They are not expected to be appreciable for large classical systems - and these quantum violations are certainly not expected to play a role in cosmological settings. (Except possibly in the Planck regime just after the big bang itself). In particular the classical singularity theorem relevant to proving the existence of the big-bang singularity uses the so-called strong energy condition (SEC) [3. pages 263-273] — and we would be rather surprised to see the SEC being violated at late times in a classical cosmological setting. (At least, we would be surprised if these violations occur outside of unusual parameter regimes such as the Planck slop $\left[k T \approx E_{\text {Planck }} \approx 10^{19} \mathrm{GeV}\right]$ or cosmological inflation $\left[k T \approx E_{G U T} \approx 10^{15}\right.$ $\mathrm{GeV}]$.)

It is therefore somewhat disturbing to realise that current observations seem to indicate that the SEC is violated rather late in the life of the universesomewhere between galaxy formation and the present time, in an epoch where the redshift is certainly less than 20 and the cosmological temperature never exceeds 60 Kelvin. I shall show this by using the energy conditions to develop simple and robust bounds for the density and look-back time as a function of red-shift in Friedmann-Robertson-Walker (FRW) cosmologies.

The experimental observations I need are the present day value of the Hubble parameter $H_{0}$, an age estimate for the age of the oldest stars in the galactic halo, and a crude estimate for the red-shift at which these oldest stars formed. From the theoretical side, I only need to use a FRW cosmology subject to the Einstein equations and the energy conditions, and nothing more.

I do not need to make a specific commitment to the use of cold, hot, lukewarm, or mixed dark matter, nor to MACHOS, WIMPS, or other exotic astroparticle contributions to the cosmological density. Avoiding the need for 
these commitments is what the energy conditions are good for. Sometimes we are even luckier: some of the key results of this paper can be phrased in a way that is independent of whether or not the universe is open, flat, or closedthis has the advantage that we can side-step the whole tangle of disagreements surrounding the $\Omega$ parameter (see also [2]), and completely avoid committing ourselves to the existence or nonexistence of any of the standard variants of cosmological inflation.

\section{Energy conditions in a FRW Universe}

\subsection{FRW cosmology}

The standard FRW cosmology is described by the metric 14, 15, 16, 17]

$$
d s^{2}=-d t^{2}+a(t)^{2}\left[\frac{d r^{2}}{1-k r^{2}}+r^{2}\left(d \theta^{2}+\sin ^{2} \theta d \phi^{2}\right)\right] .
$$

with

$$
k=\left\{\begin{array}{rc}
+1 & \text { closed } \\
0 & \text { flat } \\
-1 & \text { open }
\end{array}\right.
$$

The two non-trivial components of the Einstein equations are 14, 15, 16, 17

$$
\begin{gathered}
\rho=\frac{3}{8 \pi G}\left[\frac{\dot{a}^{2}}{a^{2}}+\frac{k}{a^{2}}\right] . \\
p=-\frac{1}{8 \pi G}\left[2 \frac{\ddot{a}}{a}+\frac{\dot{a}^{2}}{a^{2}}+\frac{k}{a^{2}}\right] .
\end{gathered}
$$

They can be combined to deduce the conservation of stress-energy 14, 15, 16, 17

$$
\dot{\rho}=-3 \frac{\dot{a}}{a}(\rho+p)
$$

\subsection{Point-wise energy conditions}

The standard point-wise energy conditions are the null energy condition (NEC), weak energy condition (WEC), strong energy condition (SEC), and dominant energy condition (DEC). Basic definitions are given for instance in 3, 10]. For the case of a FRW spacetime the definitions specialize to [1]

$$
\begin{gathered}
\mathrm{NEC} \Longleftrightarrow \quad(\rho+p \geq 0) \\
\mathrm{WEC} \Longleftrightarrow \quad(\rho \geq 0) \text { and }(\rho+p \geq 0) .
\end{gathered}
$$




$$
\begin{gathered}
\text { SEC } \Longleftrightarrow \quad(\rho+3 p \geq 0) \text { and }(\rho+p \geq 0) . \\
\text { DEC } \Longleftrightarrow \quad(\rho \geq 0) \text { and }(\rho \pm p \geq 0) .
\end{gathered}
$$

Thus in this context the energy conditions are just simple constraints on various linear combinations of the energy density and pressure. Since "normal" matter has both positive energy density and positive pressure, normal matter will automatically satisfy the NEC, WEC, and SEC. If we add the assumption that the speed of sound in normal matter is always less than that of light then in addition $\partial \rho / \partial p<1$. Assuming no cosmological constant, and suitable behaviour at $p=0$, integrating this yields $p<\rho$, so the DEC will be satisfied as well. (Roughly speaking: violating the DEC is typically associated with either a large negative cosmological constant or superluminal acoustic modes.)

That is, normal matter satisfies all the standard energy conditions and so any of the standard energy conditions can be used to place constraints on the behaviour of the universe.

I shall call matter that violates any one of the energy conditions "not normal" and shall reserve the word "abnormal" for matter that specifically violates the SEC. In keeping with prior usage [10, 18, 19], shall call matter that violates the NEC "exotic". (Thus all exotic matter is both abnormal and not normal, whereas abnormal matter need not be exotic.)

I should immediately point out that getting hold of exotic matter is intrinsically a quantum mechanical enterprise: all decent classical lagrangians satisfy the NEC and it is only by going to semi-classical quantum effects that NEC violations are ever encountered. (See, for instance, 10, 20, 21, 22, 23, 24.) On the other hand, violating the WEC and DEC is relatively easy (but not absurdly so): Violating the WEC and DEC (but not the NEC and SEC) can be accomplished with a negative cosmological constant [10, page 129-130]. Finally, violating the SEC is also relatively easy (but again not absurdly so): Violating the SEC (but not the NEC, WEC, and DEC) can be accomplished for instance, either by a positive cosmological constant or by a cosmological inflationary epoch 110, page 129-130].

Thus the basic logic of this paper is as follows: normal matter satisfies all the energy conditions and puts tight constraints on the expansion of the universe. If the universe seems to observationally violate these constraints then this gives us information about the existence of non normal matter. In particular, this gives us a handle on the existence of abnormal matter such as a cosmological constant or a cosmological inflaton field. 


\section{Density bounds from the energy conditions}

Certain elementary consequences of the energy conditions can be read off by inspection.

\section{$3.1 \quad$ NEC}

The NEC is enough to guarantee that the density of the universe goes down as its size increases.

$$
\mathrm{NEC} \Longleftrightarrow \operatorname{sign}(\dot{\rho})=-\operatorname{sign}(\dot{a}) .
$$

(This is most simply derived from the equation of stress-energy conservation, when combined with the definition of the NEC.)

If we violate the NEC the density of the universe must grow as the universe grows - so something has gone very seriously wrong. (In particular, not even a cosmological constant will let you violate the NEC.) Note that we do not even need to know if the universe is spatially open, flat, or closed to get this conclusion.

\section{$3.2 \quad$ WEC}

The WEC additionally tells us that the density is not only non-increasing, it's positive. In a FRW setting the WEC does not provide any stronger bound on the energy density as a function of scale parameter.

\subsection{SEC}

To see what the SEC does, consider the quantity $d\left(\rho a^{2}\right) / d t$, and use the Einstein equations to deduce

$$
\frac{d}{d t}\left(\rho a^{2}\right)=-a \dot{a}(\rho+3 p) .
$$

Thus

$$
\mathrm{SEC} \Rightarrow \quad \operatorname{sign}\left[\frac{d}{d t}\left(\rho a^{2}\right)\right]=-\operatorname{sign}(\dot{a}) .
$$

This implies that

$$
\mathrm{SEC} \Rightarrow \quad \rho(a) \geq \rho_{0}\left(a_{0} / a\right)^{2} \quad \text { for } \quad a<a_{0} .
$$

In terms of the red-shift

$$
\mathrm{SEC} \Rightarrow \quad \rho(z) \geq \rho_{0}(1+z)^{2} .
$$


As usual, the subscript zero denotes present day values, and the SEC is providing us with a model independent lower bound on the density as we extrapolate back to the big bang. (This bound again being independent of whether or not the universe is open, flat, or closed.)

Another viewpoint on the SEC comes from considering the quantity

$$
\rho+3 p=-\frac{3}{4 \pi G}\left[\frac{\ddot{a}}{a}\right] .
$$

That is

$$
\mathrm{SEC} \Rightarrow \quad \ddot{a}<0 .
$$

The SEC implies that the expansion of the universe is decelerating - and this conclusion holds independent of whether the universe is open, flat, or closed.

It is usual to define a deceleration parameter [14:

$$
q_{0}=-\frac{\ddot{a} a}{\dot{a}^{2}} .
$$

In which case:

$$
\mathrm{SEC} \Rightarrow \quad q_{0}>0 .
$$

In particular, any analysis of galaxy distribution that implies a negative deceleration parameter also implies, ipso facto, violations of the SEC. See for instance [25. Note that these SEC violations are all by definition going on at relatively low redshift (certainly $z<7$ ) since there are no visible galaxies beyond this range.

In terms of critical density $\left[\rho_{\text {critical }}=3 H_{0}^{2} /(8 \pi G)\right]$ we have

$$
q_{0}=\frac{1}{2} \frac{\rho+3 p}{\rho_{\text {critical }}} .
$$

\subsection{DEC}

Now turning attention to the DEC, it is useful to compute

$$
\frac{d}{d t}\left(\rho a^{6}\right)=+3 a^{5} \dot{a}(\rho-p) .
$$

Thus

$$
\mathrm{DEC} \Rightarrow \quad \operatorname{sign}\left[\frac{d}{d t}\left(\rho a^{6}\right)\right]=+\operatorname{sign}(\dot{a}) .
$$

The DEC therefore provides an upper bound on the energy density.

$$
\mathrm{DEC} \Rightarrow \quad \rho(a) \leq \rho_{0}\left(a_{0} / a\right)^{6} \quad \text { for } \quad a<a_{0} .
$$

In terms of the red-shift 


$$
\mathrm{DEC} \Rightarrow \quad \rho(z) \leq \rho_{0}(1+z)^{6}
$$

\subsection{Standard toy models}

It is instructive to compare these bounds to the standard toy models for the density: dust, radiation, and cosmological constant. Indeed

$$
\begin{aligned}
\rho_{\text {dust }}(z) & =\rho_{\text {dust }}(0)(1+z)^{3} \\
\rho_{\text {radiation }}(z) & =\rho_{\text {dust }}(0)(1+z)^{4} \\
\rho_{\Lambda}(z) & =\rho_{\Lambda}(0) .
\end{aligned}
$$

For a mixture of these three types of matter

$$
\rho(z)=\rho_{\text {critical }}\left[\Omega_{\text {dust }}(1+z)^{3}+\Omega_{\text {radiation }}(1+z)^{4}+\Omega_{\Lambda}\right] .
$$

This serves as the definition of the partial $\Omega$ parameters: $\Omega_{\text {dust }}, \Omega_{\text {radiation }}$, and $\Omega_{\Lambda}$. Additionally, of course, we have

$$
\Omega=\Omega_{\text {dust }}+\Omega_{\text {radiation }}+\Omega_{\Lambda} .
$$

Note that $(\rho+3 p)_{\Lambda}=-2 \rho_{\Lambda}$, while $(\rho+3 p)_{\text {dust }}=\rho_{\text {dust }}$, and $(\rho+3 p)_{\text {radiation }}=$ $+2 \rho_{\text {radiation }}$. Thus, if one restricts oneself to mixtures of these three types of matter, the deceleration parameter can be written as

$$
q_{0}=-\Omega_{\Lambda}+\frac{1}{2} \Omega_{\text {dust }}+\Omega_{\text {radiation }}
$$




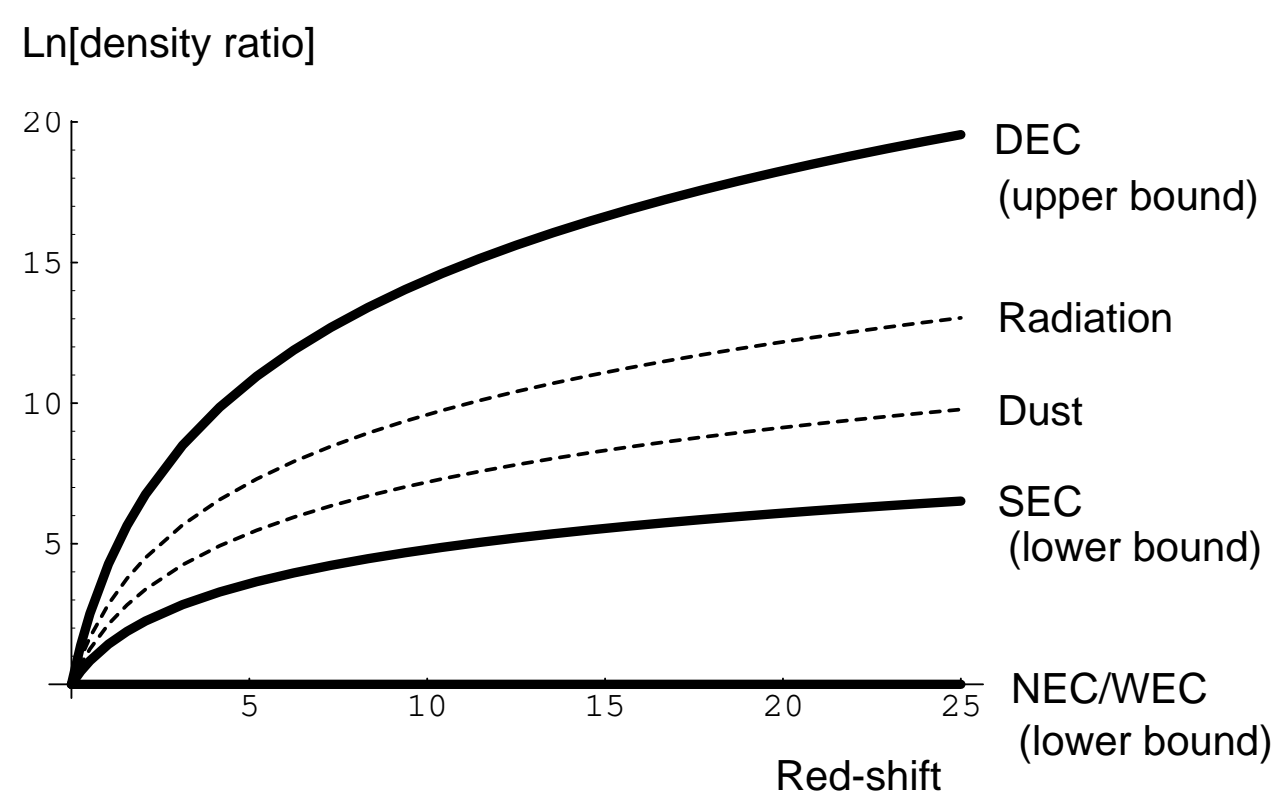

Figure 1: Model independent bounds on the density of the universe as a function of redshift. Curves are plotted for the NEC, SEC, and DEC from redshift zero (here and now) to redshift 25 (comfortably prior to galaxy formation). For the purposes of this calculation the WEC and NEC are indistinguishable. For comparison, dotted curves are added for pure dust and for pure radiation. The curve corresponding to pure cosmological constant lies directly on top of that for the NEC. 


\section{Look-back time- some bounds}

Particularly useful results can be obtained when we consider the look-back time as a function of red-shift. The look-back time, $\tau=\left|t-t_{0}\right|$, is the difference between the age of the universe when a particular light ray was emitted and the age of the universe now as we are receiving it. If we know the velocity of expansion $\dot{a}$ as a function of size $a$ we have

$$
\tau\left(a ; a_{0}\right)=\left|t-t_{0}\right|=\int_{a}^{a_{0}} \frac{d a}{\dot{a}(a)} .
$$

By putting a lower bound on $\dot{a}$ we deduce an upper bound on look-back time, whereas from an upper bound on $\dot{a}$ we deduce a lower bound on look-back time.

\subsection{SEC}

Since the SEC implies that the expansion is decelerating, we immediately see

$$
\mathrm{SEC} \Rightarrow \quad \tau\left(a ; a_{0}\right) \leq \frac{a-a_{0}}{\dot{a}\left(a_{0}\right)} .
$$

Thus

$$
\mathrm{SEC} \Rightarrow \quad \tau\left(a ; a_{0}\right)=\left|t-t_{0}\right| \leq \frac{1}{H_{0}} \frac{a_{0}-a}{a_{0}},
$$

this relation being independent of whether the universe is open, flat, or closed. Expressed in terms of the red-shift:

$$
\mathrm{SEC} \Rightarrow \quad \tau(z)=\left|t-t_{0}\right| \leq \frac{1}{H_{0}} \frac{z}{1+z} \leq \frac{1}{H_{0}} .
$$

This provides us with a robust upper bound on the Hubble parameter

$$
\mathrm{SEC} \Rightarrow \quad \forall z: H_{0} \leq \frac{1}{\tau(z)} \frac{z}{1+z} \leq \frac{1}{\tau(z)} .
$$

\section{2 $\quad \mathrm{NEC}(\mathrm{k}=0)$}

In contrast, when we look at the NEC, we do not get a constraint that is independent of spatial curvature. For a spatially flat universe $(k=0$, as preferred by the inflation advocates), we easily see that $\dot{a} / a \equiv H(a) \geq H_{0}$. (Here $t \leq t_{0}$, $a \leq a_{0}$.) Integrating this constraint yields

$$
\mathrm{NEC}+(k=0) \Rightarrow \quad \ln \left(a_{0} / a\right) \leq H_{0}\left(t_{0}-t\right) .
$$

In terms of the red-shift 


$$
\begin{gathered}
\mathrm{NEC}+(k=0) \Rightarrow \quad \tau=\left|t-t_{0}\right| \leq \frac{\ln (1+z)}{H_{0}} . \\
\mathrm{NEC}+(k=0) \Rightarrow \quad \forall z: H_{0} \leq \frac{\ln (1+z)}{\tau(z)} .
\end{gathered}
$$

Somewhat messier formulae are derived below for $k= \pm 1$.

\subsection{DEC $(\mathrm{k}=0)$}

Finally, we turn to the DEC: As we have seen, this energy condition provides us with a upper bound on the energy density, and therefore an upper bound on the rate of expansion. This translates to a lower bound on the look-back time. For a spatially flat universe ( $k=0$, as preferred by the inflation advocates) the previously derived density bound quickly yields

$$
\mathrm{DEC}+(k=0) \Rightarrow\left(\frac{\dot{a}}{a}\right)^{2} \leq H_{0}^{2}\left(\frac{a_{0}}{a}\right)^{6} .
$$

Integrating this constraint

$$
\mathrm{DEC}+(k=0) \Rightarrow \quad \tau=\left|t-t_{0}\right| \geq \frac{1}{3 H_{0}} \frac{a_{0}^{3}-a^{3}}{a_{0}^{3}} \leq \frac{1}{3 H_{0}} .
$$

Note that the direction of this last inequality implies that in this case one cannot get a simple bound on $\tau$ merely in terms of $H_{0}$, some extra information is needed. In terms of the red-shift

$$
\begin{gathered}
\mathrm{DEC}+(k=0) \Rightarrow \quad \tau=\left|t-t_{0}\right| \geq \frac{1}{3 H_{0}}\left(1-\frac{1}{(1+z)^{3}}\right) \leq \frac{1}{3 H_{0}} . \\
\mathrm{DEC}+(k=0) \Rightarrow \quad \forall z: H_{0} \geq \frac{1}{3 \tau(z)}\left(1-\frac{1}{(1+z)^{3}}\right) \leq \frac{1}{3 \tau(z)} .
\end{gathered}
$$
then

If we can estimate $\tau(\infty)$ - the lookback tine all the way to the big bang-

$$
\mathrm{DEC}+(k=0) \Rightarrow \quad H_{0} \geq \frac{1}{3 \tau(\infty)} .
$$

Somewhat messier formulae are derived below for $k= \pm 1$.

\subsection{Some benchmarks}

For completeness and benchmarking I mention here some standard results. 


\subsubsection{Dust}

$$
\begin{aligned}
& \text { Dust }+(k=0) \Rightarrow \quad \tau=\left|t-t_{0}\right|=\frac{2}{3 H_{0}}\left(1-\frac{1}{(1+z)^{3 / 2}}\right) \leq \frac{2}{3 H_{0}} \\
& \text { Dust }+(k=0) \Rightarrow \quad \forall z: H_{0}=\frac{2}{3 \tau(z)}\left(1-\frac{1}{(1+z)^{3 / 2}}\right) \leq \frac{2}{3 \tau(z)}
\end{aligned}
$$

\subsubsection{Radiation}

$$
\begin{aligned}
& \text { Radiation }+(k=0) \Rightarrow \quad \tau=\left|t-t_{0}\right|=\frac{1}{2 H_{0}}\left(1-\frac{1}{(1+z)^{2}}\right) \leq \frac{1}{2 H_{0}} \\
& \text { Radiation }+(k=0) \Rightarrow \quad \forall z: H_{0}=\frac{1}{2 \tau(z)}\left(1-\frac{1}{(1+z)^{2}}\right) \leq \frac{1}{2 \tau(z)}
\end{aligned}
$$

\subsubsection{Linear fluid}

Define a linear fluid by the equation of state $p=\gamma \rho$ with $\rho>0$ and $\gamma \in[-1,+1]$ so as to satisfy the DEC. The SEC is still violated over the range $\gamma \in[-1,-1 / 3)$, whereas the fluid is normal in the range $\gamma \in[-1 / 3,+1]$.

$$
\text { Linear fluid } \Rightarrow \quad \rho=\rho_{0}(1+z)^{3(1+\gamma)}
$$

$$
\begin{aligned}
& \text { Linear fluid }+(k=0) \Rightarrow \\
& \qquad \tau=\left|t-t_{0}\right|=\frac{2}{3(1+\gamma) H_{0}}\left(1-\frac{1}{(1+z)^{3(1+\gamma) / 2}}\right) \leq \frac{2}{3(1+\gamma) H_{0}}
\end{aligned}
$$

$$
\begin{aligned}
& \text { Linear fluid }+(k=0) \Rightarrow \quad \forall z: \\
& \qquad H_{0}=\frac{2}{3(1+\gamma) \tau(z)}\left(1-\frac{1}{(1+z)^{3(1+\gamma) / 2}}\right) \leq \frac{2}{3(1+\gamma) \tau(z)}
\end{aligned}
$$

Special cases: $\gamma=-1$ is a cosmological constant, and for suitable choices of the other cosmological parameters can lead to a "hesitation universe" as discussed in MTW [15, pages 746-747]. The range $\gamma \in(-1,-1 / 3)$ corresponds to the "loitering universe" 26, 27, the point $\gamma=-1 / 3$ is relevant to the "lowdensity closed universe" scenario [28, while the range $\gamma \in(-1,0)$ corresponds to the so-called "decaying cosmological constant" 29, 30. 


\subsection{4 de Sitter}

(Pure cosmological constant, no other matter.)

$$
\begin{gathered}
\text { de Sitter }+(k=0) \Rightarrow \quad \tau=\left|t-t_{0}\right|=\frac{\ln (1+z)}{H_{0}} . \\
\text { de Sitter }+(k=0) \Rightarrow \quad \forall z: H_{0}=\frac{\ln (1+z)}{\tau(z)} .
\end{gathered}
$$

\subsubsection{Milne}

The Milne universe is an interesting non-standard cosmology [14, page 198-199]. It is actually a segment of Minkowski space in disguise, obtained by picking a point in Minkowski space, taking the future event cone of that point, and then placing a FRW metric on this cone. The Milne universe is intrinsically open $(k=-1)$ and has the metric

$$
d s^{2}=-d t^{2}+t^{2}\left[\frac{d r^{2}}{1+r^{2}}+r^{2}\left(d \theta^{2}+\sin ^{2} \theta d \phi^{2}\right)\right] .
$$

It is now trivial to see

$$
\begin{gathered}
\text { Milne } \Rightarrow \quad \tau\left(a ; a_{0}\right)=\left|t-t_{0}\right|=\frac{1}{H_{0}} \frac{a_{0}-a}{a_{0}}, \\
\text { Milne } \Rightarrow \quad \forall z: H_{0}=\frac{1}{\tau(z)} \frac{z}{1+z} \leq \frac{1}{\tau(z)} .
\end{gathered}
$$

Thus the Milne universe is an example of a cosmological model that exactly saturates the SEC bound - of course it does so for a trivial reason: Since the Milne universe is a segment of Minkowski space it is clearly empty vacuum, so $\rho+3 p=0$ for the simple reason that both $\rho$ and $p$ are individually zero.

To make an empty Milne universe compatible with the observed existence of galaxies one has, at a minimum, to demand a fractal distribution for the galaxies, so that the average density of galaxies (averaged over sufficiently large regions) converges to zero as the size of the averaging region increases. To fit observational data, this has to be (at the very least) a multi-fractal structure with strong evolutionary effects. (See Peebles [14, pages 209-224].) This still leaves one with the issue of the cosmic microwave background (CBR), is it to be taken to be uniform or multifractal? In short, the Milne universe is not interesting because it is a good fit to the data, rather it is interesting because it is a useful benchmark to compare other models to. 


\subsection{Some somewhat messier formulae}

\subsubsection{NEC $(k= \pm 1)$}

If the spatial curvature is not zero $(k= \pm 1)$ the NEC inspired constraint on the density is more complicated. For $t<t_{0}$ we have

$$
\left[\frac{\dot{a}^{2}}{a^{2}}+\frac{k}{a^{2}}\right] \geq\left[\frac{\dot{a}_{0}^{2}}{a_{0}^{2}}+\frac{k}{a_{0}^{2}}\right] \equiv \Omega H_{0}^{2} .
$$

Note that $k$ and $\Omega$ cannot be chosen independently. In fact by definition

$$
H_{0}^{2}(\Omega-1)=\frac{k}{a_{0}^{2}}
$$

so that $k=\operatorname{sign}(\Omega-1)$.

The above inequality is easily rearranged to give

$$
\dot{a}^{2} \geq \Omega H_{0}^{2} a^{2}-k .
$$

More properly, $\dot{a}^{2} \geq \max \left\{\Omega H_{0}^{2} a^{2}-k, 0\right\}$. From the definition of look-back time

$$
\tau\left(a_{0} ; a\right) \leq \int_{a}^{a_{0}} \frac{d a}{\sqrt{\Omega H_{0}^{2} a^{2}-k}}
$$

(If $k=+1$ the argument of the square root will eventually go imaginary for small enough $a$ [large enough redshift]. This just means that the bound is so weak $[\dot{a}>0]$ as to be useless once we go too far back [beyond $\left(1+z_{\text {limit }}\right)^{2}=$ $\Omega /(\Omega-1)]$.)

Integrating, I find

$$
\tau\left(a_{0} ; a\right) \leq \frac{1}{\sqrt{\Omega} H_{0}} \ln \left[\frac{\sqrt{\Omega H_{0}^{2} a_{0}^{2}}+\sqrt{\Omega H_{0}^{2} a_{0}^{2}-k}}{\sqrt{\Omega H_{0}^{2} a^{2}}+\sqrt{\Omega H_{0}^{2} a^{2}-k}}\right] .
$$

Equivalently

$$
\tau\left(a_{0} ; a\right) \leq \frac{1}{\sqrt{\Omega} H_{0}} \ln \left[\frac{a_{0}(\sqrt{\Omega}+1)}{a \sqrt{\Omega}+\sqrt{a^{2} \Omega-a_{0}^{2}(\Omega-1)}}\right] .
$$

(I have now assumed that $\Omega>0$, so that the WEC is satisfied, density is positive, and the appropriate square roots are real.)

For $k=0(\Omega=1)$ this reproduces the results previously discussed.

For general $k$, if we cast this result in terms of the redshift

$$
\mathrm{WEC} \Rightarrow \quad \tau(z) \leq \frac{1}{\sqrt{\Omega} H_{0}} \ln \left[(1+z)\left(\frac{\sqrt{\Omega}+1}{\sqrt{\Omega}+\sqrt{1-(\Omega-1)\left[(1+z)^{2}-1\right]}}\right)\right]
$$


Thus the analytic bound on the Hubble parameter is

$$
\begin{aligned}
\mathrm{WEC} & \Rightarrow \quad \forall z: \\
H_{0} & \leq \frac{1}{\sqrt{\Omega} \tau(z)} \ln \left[(1+z)\left(\frac{\sqrt{\Omega}+1}{\sqrt{\Omega}+\sqrt{1-(\Omega-1)\left[(1+z)^{2}-1\right]}}\right)\right] .
\end{aligned}
$$

Finally, for $\Omega$ close to 1, we can Taylor expand this general formula to yield

$$
\begin{aligned}
& \mathrm{WEC} \Rightarrow \quad \forall z: \\
& H_{0} \leq \frac{1}{\sqrt{\Omega} \tau(z)}\left[\ln (1+z)+\frac{(\sqrt{\Omega}-1)\left[(1+z)^{2}-1\right]}{2}+O\left[(\sqrt{\Omega}-1)^{2}\right]\right] .
\end{aligned}
$$

Turning now to some special cases, if $k=-1$ (so that $\Omega<1$; remember that we already assumed $\Omega>0$ to satisfy the WEC) equation (61) may be rewritten in the somewhat simpler but still rather clumsy form

$$
\begin{aligned}
& \mathrm{WEC}+(k=-1) \Rightarrow \quad \forall z: \\
& \tau(z) \leq \frac{1}{\sqrt{\Omega} H_{0}}\left[\sinh ^{-1}\left(\sqrt{\Omega} H_{0} a_{0}\right)-\sinh ^{-1}\left(\frac{\sqrt{\Omega} H_{0} a_{0}}{1+z}\right)\right] .
\end{aligned}
$$

Equivalently

$$
\begin{aligned}
& \mathrm{WEC}+(k=-1) \Rightarrow \quad \forall z: \\
& \tau(z) \leq \frac{1}{\sqrt{\Omega} H_{0}}\left[\sinh ^{-1}\left(\sqrt{H_{0}^{2} a_{0}^{2}-1}\right)-\sinh ^{-1}\left(\frac{\sqrt{H_{0}^{2} a_{0}^{2}-1}}{1+z}\right)\right] .
\end{aligned}
$$

(The square roots are real because I have used the WEC to constrain $\Omega>0$.)

The most analytically transparent form of the inequality for $k=-1$ follows by noting that the term in large brackets in (61) and (62) is less than 1 (for $k=-1, \Omega<1, \Omega>0$, and $z>0$ ). Consequently its logarithm is negative and we have the somewhat weaker (but analytically much more tractable) bound that

$$
\mathrm{WEC}+(k=-1) \Rightarrow \quad \tau(z) \leq \frac{1}{\sqrt{\Omega} H_{0}} \ln [1+z]
$$




$$
\mathrm{WEC}+(k=-1) \Rightarrow \quad \forall z: H_{0} \leq \frac{1}{\sqrt{\Omega} \tau(z)} \ln [1+z] .
$$

We could also get this more directly from the inequality (valid only for $k=-1$ !)

$$
\left[\frac{\dot{a}^{2}}{a^{2}}\right] \geq\left[\frac{\dot{a}^{2}}{a^{2}}+\frac{k}{a^{2}}\right] \geq \Omega H_{0}^{2} .
$$

For $k=+1$ no nice simplification takes place and one must retain the full complexity of equations (61), (62) and (63).

\subsubsection{DEC $(k= \pm 1)$}

If the spatial curvature is not zero $(k= \pm 1)$ the DEC inspired constraint on the density becomes, for $t<t_{0}$,

$$
\left[\frac{\dot{a}^{2}}{a^{2}}+\frac{k}{a^{2}}\right] a^{6} \leq\left[\frac{\dot{a}_{0}^{2}}{a_{0}^{2}}+\frac{k}{a_{0}^{2}}\right] a_{0}^{6} \equiv \Omega H_{0}^{2} a_{0}^{6} .
$$

This is easily rearranged to give

$$
\dot{a}^{2} \leq \frac{\Omega H_{0}^{2} a_{0}^{6}}{a^{4}}-k .
$$

From the definition of look-back time

$$
\tau\left(a_{0} ; a\right) \geq \int_{a}^{a_{0}} \frac{a^{2} d a}{\sqrt{\Omega H_{0}^{2} a_{0}^{6}-k a^{4}}} .
$$

(Provided $\Omega>0$, which is automatically implied by the DEC, the argument of the square root will always be positive.)

For $k= \pm 1$ this integral is expressible as an elliptic integral plus elementary functions - the form is not particularly illuminating. A slightly more transparent form follows from using the relation $k=H_{0}^{2} a_{0}^{2}(\Omega-1)$ to write

$$
\tau\left(a_{0} ; a\right) \geq \frac{1}{\sqrt{\Omega} H_{0}} \int_{a}^{a_{0}} \frac{a^{2} d a}{a_{0}^{3} \sqrt{1-\frac{\Omega-1}{\Omega}\left(\frac{a^{4}}{a_{0}^{4}}\right)}} .
$$

This representation admits a power series expansion around flat space $(\Omega=1)$. Taylor series expanding the integrand, integrating, and summing, yields

$$
\mathrm{DEC} \Rightarrow \quad \tau\left(a_{0} ; a\right) \geq \frac{1}{\sqrt{\Omega} H_{0}} \sum_{n=0}^{\infty}\left(\begin{array}{c}
\frac{1}{2} \\
n
\end{array}\right)\left(\frac{\Omega-1}{\Omega}\right)^{n} \frac{1}{3+4 n}\left[1-\left(\frac{a}{a_{0}}\right)^{3+4 n}\right] .
$$


The most analytically transparent form of the inequality for $k=+1$ follows by noting that the square root in (72) is less than 1 (for $k=+1, \Omega>1$ ). Consequently we have the somewhat weaker (but analytically much more tractable) bound that

$$
\mathrm{DEC}+(k=+1) \Rightarrow \quad \tau\left(a_{0} ; a\right) \geq \frac{1}{\sqrt{\Omega} 3 H_{0}}\left[1-\left(\frac{a}{a_{0}}\right)^{3}\right] .
$$

For $k=-1$ no such nice simplification takes place and one must retain the full complexity of equation (73).

\section{The "age-of-the-oldest-stars" problem}

\subsection{SEC}

The bound derived from the SEC is enough to illustrate the "age-of-the-oldeststars" problem. Suppose we have some class of standard candles whose age of formation, $\tau_{f}$, we can by some means estimate. Suppose further that we look out far enough can see some of these standard candles forming at red-shift $z_{f}$. Then

$$
\mathrm{SEC} \Rightarrow \quad H_{0} \leq \frac{1}{\tau_{f}} \frac{z_{f}}{1+z_{f}} .
$$

The standard candles most of interest are the globular clusters in the halos of spiral galaxies: stellar evolution models lead to the estimate (not a measurement!) that the age of oldest stars still extant is $16 \pm 2 \times 10^{9}$ yr [14, page 106]. That is, at an absolute minimum

$$
\text { Age of oldest stars } \equiv \tau_{f} \geq 16 \pm 2 \times 10^{9} \mathrm{yr} .
$$

Using $z_{f}<\infty$, this immediately implies

$$
H_{0}\left(z_{f}<\infty\right) \leq \tau_{f}^{-1} \leq 62 \pm 8 \mathrm{~km} \mathrm{~s}^{-1} \mathrm{Mpc}^{-1} .
$$

This bound is already of some concern, and will now be somewhat tightened.

When we actually look into the night sky, the galaxies appear to be forming at redshifts out to $z \approx 4-5$. The oldest stars, which we cannot see forming directly, are inferred to be forming somewhat earlier than the development of galactic spiral structure. There is considerable uncertainty and model dependence in the estimates of the redshift at formation, but fortunately the analysis below is not particularly sensitive to the precise numbers used. (I emphasize that making the redshift of formation smaller will make the bounds more stringent, not looser, and will make the age problem worse, not better.) A canonical estimate is 14, page 614] 


$$
\text { Redshift at formation of oldest stars } \equiv z_{f} \approx 15 .
$$

This now bounds the Hubble parameter

$$
\mathrm{SEC} \Rightarrow \quad H_{0}\left(z_{f} \approx 15\right) \leq 58 \pm 7 \mathrm{~km} \mathrm{~s}^{-1} \mathrm{Mpc}^{-1} .
$$

If we reduce $z_{f}$ to be more in line with the formation of the rest of the galactic structure (say $z_{f} \approx 7$ ) we make the bound more stringent

$$
\mathrm{SEC} \Rightarrow \quad H_{0}\left(z_{f} \approx 7\right) \leq 54 \pm 7 \mathrm{~km} \mathrm{~s}^{-1} \mathrm{Mpc}^{-1} .
$$

In the other direction, if we push the red-shift at formation $z_{f}$ out to its maximum reasonable value $z_{f} \approx 20$ [14, page 611], we only gain a trivial relaxation of the bound on the Hubble parameter.

$$
\mathrm{SEC} \Rightarrow \quad H_{0}\left(z_{f} \approx 20\right) \leq 59 \pm 8 \mathrm{~km} \mathrm{~s}^{-1} \mathrm{Mpc}^{-1} .
$$

These bounds are presented graphically in figures 2 and 3 .

These bounds should be compared with recent estimates of the present day value of the Hubble parameter are [31]

$$
H_{0} \in(65,85) \mathrm{km} \mathrm{s}^{-1} \mathrm{Mpc}^{-1} \text {. }
$$

(Note that I prefer not to ambulance-chase by picking the latest contentious estimate, but instead am using a range on which there is wide-spread though not universal consensus.)

But even the lowest reasonable value for the Hubble parameter $\left(H_{0}=65\right.$ $\mathrm{km} \mathrm{s}^{-1} \mathrm{Mpc}^{-1}$ ) is only just barely compatible with the SEC, and that only by taking the lowest reasonable value for the age of the globular clusters. For currently favored values of the Hubble parameter we deduce that the SEC must be violated somewhere between the formation of the oldest stars and the present time.

Note the qualifications that should be attached to this claim: We have to rely on stellar structure calculations (for $\tau_{f}$ ), an estimate for the red-shift at formation, and consensus values for the Hubble parameter.

Depending on one's reaction to this state of affairs there are three distinct camps one might wish to choose: The first camp wants to patch things up by reducing the Hubble parameter, the second by reducing the age of the oldest stars. Many theorists belong to the third camp and are quite happy with a cosmologically significant cosmological constant.

Note that all these difficulties are occurring at low cosmological temperatures $(T \leq 60 \mathrm{~K})$, and late times $(z<20)$, in a region where we thought we understood the basic equation of state of the cosmological fluid (dust!). 


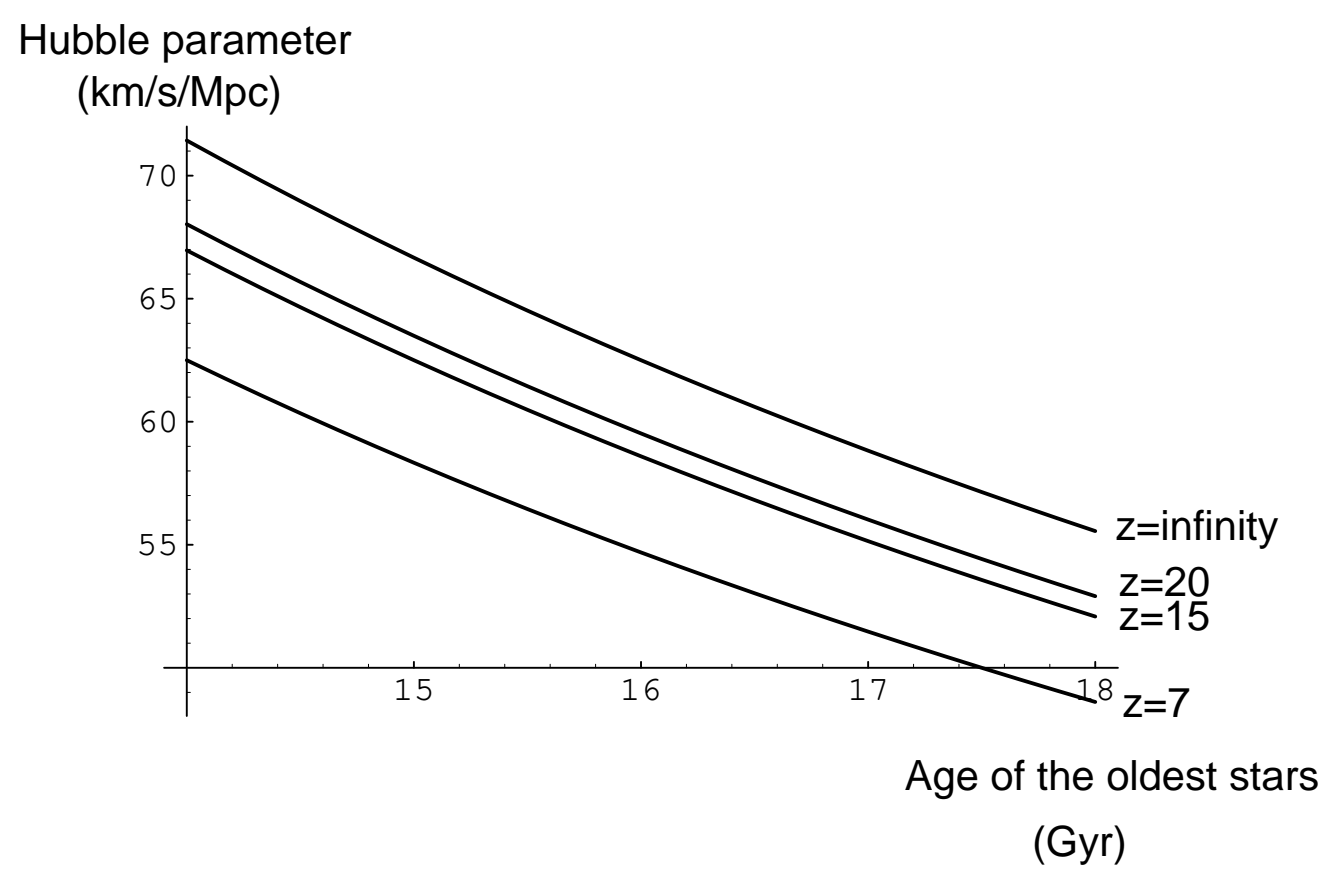

Figure 2: Model independent upper bounds on the Hubble parameter as a function of the lookback time for various assumed redshifts of formation. Curves are plotted for the upper bound derived from the SEC for redshift 7, 15, 20, and $\infty$. This plot concentrates on the most likely region for the age of the oldest stars $16 \pm 2$ Gyr. 


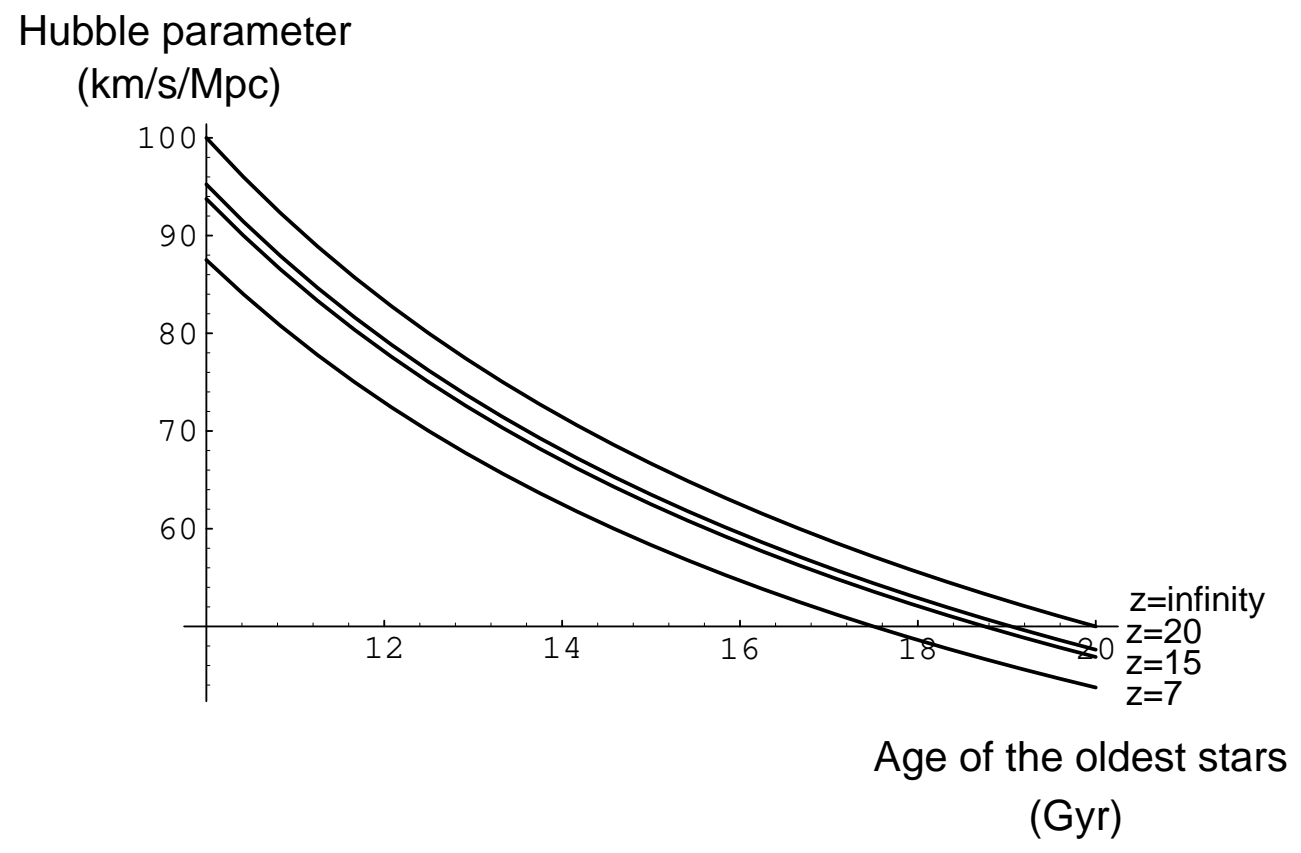

Figure 3: Model independent upper bounds on the Hubble parameter as a function of the lookback time for various assumed redshifts of formation. Curves are plotted for the upper bound derived from the SEC for redshift 7, 15, 20, and $\infty$. This plot is very generous with the age of the oldest stars: $15 \pm 5$ Gyr. 


\section{$5.2 \quad \mathrm{NEC}$}

The NEC implies a (very weak) upper bound on the Hubble parameter. In order for cosmological expansion to be compatible with stellar evolution and the NEC

$$
\mathrm{NEC}+(k=0) \Rightarrow \quad H_{0} \leq \frac{\ln \left(1+z_{f}\right)}{\tau_{f}} .
$$

The central value for $\tau_{f}$ (16 Gyr), and best guess for $z_{f}\left(z_{f} \approx 15\right)$, gives $H_{0} \leq 170 \mathrm{~km} \mathrm{~s}^{-1} \mathrm{Mpc}^{-1}$. Pulling $z_{f}$ in to $z_{f} \approx 7$ reduces this bound slightly to $H_{0} \leq 129 \mathrm{~km} \mathrm{~s}^{-1} \mathrm{Mpc}^{-1}$. Even for a high Hubble parameter $\left(H_{0}=85 \mathrm{~km} \mathrm{~s}^{-1}\right.$ $\left.\mathrm{Mpc}^{-1}\right)$, and high age for the oldest stars $\left(\tau_{f}=18 \times 10^{9} \mathrm{yr}\right)$, we only obtain the quite reasonable constraint $z_{f} \geq 3.6$. The present data is not in conflict with the NEC and in fact leaves us with considerable maneuvering room. The good news is that this bound at least tells us we are down to arguing over the last factor of four, and that we do not have any more factors of 10 hiding in the woodwork.

(The violations of the SEC discussed previously are somewhat surprising, but as we shall soon enough see, not disastrous. A potential violation of the NEC would however be very disturbing.)

\subsection{DEC}

The DEC also supplies a relatively weak constraint, this time a lower bound.

$$
\mathrm{DEC}+(k=0) \Rightarrow \quad H_{0} \geq \frac{1}{3 \tau\left(z_{f}\right)}\left(1-\frac{1}{\left(1+z_{f}\right)^{3}}\right) .
$$

Putting in the numbers we see

$$
\mathrm{DEC}+(k=0) \Rightarrow \quad H_{0} \geq 20 \pm 3 \mathrm{~km} \mathrm{~s}^{-1} \mathrm{Mpc}^{-1} .
$$

The present observational data is not in conflict with the DEC and again leaves us with considerable maneuvering room. It is only the SEC that is at all problematic. This does however tell us that we cannot go too low with our estimates for the Hubble parameter. Violating this bound would require a cosmologically large and negative cosmological constant or superluminal acoustic modes.

Some theoretical models of CBR structure now quote a Hubble parameter of order $30 \mathrm{~km} \mathrm{~s}^{-1} \mathrm{Mpc}^{-1}$, so this lower bound, though weak, is perhaps not totally lacking in interest.

Reducing the age of the oldest stars will make the upper bound on the Hubble (coming from the SEC) weaker, but will make this lower bound (from the DEC) stronger. 


\section{SEC violations-Implications}

How seriously should we take these SEC violations? There are two issues: (1) How seriously do we take the data?, and (2) What do the apparent violations of the SEC imply?

The data on the Hubble constant have historically exhibited considerable flexibility: While it is clear that the relationship between the distance and redshift is essentially linear, the absolute calibration of the slope has varied by more than an order of magnitude over the course of this century. Numbers from $630 \mathrm{~km} \mathrm{~s}^{-1} \mathrm{Mpc}^{-1}$ to $25 \mathrm{~km} \mathrm{~s}^{-1} \mathrm{Mpc}^{-1}$ can be found in the published literature 14]. Current measurements give credence to the broad range $65-85$ $\mathrm{km} \mathrm{s}^{-1} \mathrm{Mpc}^{-1}$ [31], but refinement beyond this point is uncertain.

The reliability of the data on $\tau_{f}$ and $z_{f}$ is harder to quantify, but there appears to be broad consensus within the community on these values. Note that we can re-do the analysis of this paper for any set of standard candles whose formation we think we understand.

If we accept that the SEC is violated between the epoch of galaxy formation and the present, we should ask how serious a problem this is. The two favorite ways of putting SEC violations into a classical field theory are via a massive scalar field [3, page 95], or via a positive cosmological constant [3, 10].

A classical scalar field (provided it is either massive or self interacting) can violate the SEC [3], but not the NEC, WEC, and DEC [10, page 120]. Indeed

$$
\left.(\rho+3 p)\right|_{\varphi}=\dot{\varphi}^{2}-V(\varphi) .
$$

It is this potential violation of the SEC that makes cosmological scalar fields so attractive to advocates of inflation [32, 33, 34]. In the present context, using a massive scalar field to deal with the age-of-the-oldest-stars problem is tantamount to asserting that a last dying gasp of inflation took place as the galaxies were being formed. (Hyper-weak extended late-time cosmological inflation?) This would be extremely surprising. Standard variants of inflation are driven by GUT-scale (grand unified theory) phase transitions in the early universe and take place when energies are of order $k T \approx 10^{14} \mathrm{GeV}$, (see [32]) with temperatures of order $T \approx 10^{27} \mathrm{~K}$ whereas, as we have seen, galaxy formation takes place for $T \leq 60 \mathrm{~K}$. In particular, one now has to worry about keeping inflation alive throughout Helium burning and decoupling without disturbing these events. Even more ad hoc is switching normal cosmological inflation off in the usual manner and then appealing to a second bout of inflation whose onset is after decoupling and continues into the epoch of galaxy formation. A somewhat related model is the "late-time phase transition" scenario [35].

In contrast, the current favorite fix for the age-of-the-oldest-stars problem is to introduce a positive cosmological constant, in which case

$$
(\rho+3 p)_{\text {total }}=(\rho+3 p)_{\text {normal }}-2 \rho_{\Lambda} .
$$


The observed SEC violations then imply

$$
\rho_{\Lambda} \geq \frac{1}{2}(\rho+3 p)_{\text {normal }} .
$$

Under the very mild constraint that the pressure due to normal matter in the present epoch is non-negative, we immediately deduce that more that $33 \%$ of the present-day energy density is due to a cosmological constant-and we do not need to know the equation of state of the normal component of matter to extract this result.

(Of course, if we know - or assume - a particular equation of state we can make more precise estimates: The main point of this paper is that even without committing oneself to a particular equation of state we can still make cosmologically interesting deductions.)

\section{Discussion}

In this paper I have presented an analysis of the age-of-the-oldest-stars problem that is, as far as possible, model independent. I have shown that high values of the Hubble parameter imply that the so-called strong energy condition (SEC) must be violated sometime between the epoch of galaxy formation and the present. This implies that the age-of-the-oldest-stars problem cannot simply be fixed by adjusting the equation of state of the cosmological fluid. Since all normal matter satisfies the SEC, fixing the age-of-the-oldest-stars problem will inescapably require the introduction of "abnormal" matter - indeed we will need large quantities of abnormal matter, sufficient to overwhelm the gravitational effects of the normal matter. It is this that I view as the central result of this paper.

The trade-off I have made in this paper is to sacrifice precision in the interests of robustness: The numerical bounds on the Hubble parameter are less stringent than they might otherwise have been if a particular equation of state had been chosen. I have tried to make the bounds discussed in this paper as model independent as possible. Several key results are completely independent of the $\Omega$ parameter, and are also independent of astroparticle physics assumptions such as the nature and existence of dark matter, monopoles, MACHOS, and WIMPS.

If we view the violation of the SEC as arising from a positive cosmological constant then it must be of the same order of magnitude as the normal contributions to the energy density. This implies that we are living at a special time in the evolution of the universe: We are living at (or reasonably close to) the switchover from universe dominated by matter (or matter plus radiation) to a a universe dominated by a cosmological constant. As we look to our future the universe should asymptotically approach a de Sitter universe with an exponential expansion being driven by this non-zero cosmological constant. 
A final comment regarding the big-bang singularity: The classical singularity theorems of general relativity show that SEC is a sufficient (but not necessary) condition for the existence of the big-bang singularity [3]. The SEC is not necessary for the existence of the big-bang singularity, unfortunately it is necessary for the general theorems used to guarantee the big-bang singularity. (It is relatively easy to find and exhibit explicit "bounce" geometries that satisfy all of the usual energy conditions except the SEC.) The loss of the global applicability of the SEC leaves us in the uncomfortable position of having to decide on a particular equation of state for the early universe before we can say whether or not the universe arose from a singularity or a bounce - the loss of the SEC implies loss of the general theorems. The extent to which one can rescue this situation by looking for improved theorems using different energy conditions is as yet obscure.

\section{Acknowledgements}

This research was supported by the U.S. Department of Energy.

\section{References}

[1] M. Visser, Energy conditions in the epoch of galaxy formation, Science $\mathbf{2 7 6}$ (4 April 1997) 88-90.

[2] S. Leonard and K. Lake, The Tinsley Diagram Revisited, The Astrophysical Journal, 441, (1995) L55-L56.

[3] S. W. Hawking and G. F. R. Ellis, The large scale structure of space-time, (Cambridge, England, 1973).

[4] J.L Friedmann, K. Schleich and D.M. Witt, Phys. Rev. Lett. 71, 1486 (1993); gr-qc/9305017.

[5] R. Schoen and S.T. Yau, Phys. Rev. Lett. 43 (1979) 1457-1459.

[6] R. Schoen and S.T. Yau, Commun. Mat. Phys. 65 (1979) 45-76.

[7] R. Schoen and S.T. Yau, Phys. Rev. Lett. 48 (1982) 369-371.

[8] R. Penrose, R. D. Sorkin, and E. Woolgar, (1993), gr-qc/9301015.

[9] G.L. Naber, Spacetime and Singularities: An Introduction, (Cambridge University press, 1988).

[10] M. Visser, Lorentzian wormholes - from Einstein to Hawking, (AIP Press, New York, 1995). 
[11] N. D. Birrell and P. C. W. Davies, Quantum fields in curved spacetime, (Cambridge, England, 1982).

[12] S. A. Fulling, Aspects of quantum field theory in curved space-time, (Cambridge, England, 1989).

[13] Ya. B. Zel'dovich and I. D. Novikov, Relativistic Astrophysics. Volume 2: The structure and evolution of the universe, (University of Chicago Press, 1983). See the discussion on pages 589, 590 concerning the breakdown of the energy conditions during periods of cosmologically-induced particle production. These are test-field limit calculations where the expansion of the universe generates a contribution to the stress tensor that violates the energy conditions, but back-reaction is not addressed.

[14] P. J. E. Peebles, Principles of Physical Cosmology, (Princeton University Press, 1993).

[15] C. W. Misner, K. S. Thorne, and J. A. Wheeler, Gravitation (W. H. Freeman and Company, San Francisco, 1973).

[16] R. M. Wald, General Relativity (University of Chicago Press, Chicago, 1984).

[17] S. Weinberg, Gravitation and Cosmology (Wiley, New York, 1972).

[18] M.S. Morris and K.S. Thorne, Am. J. Phys. 56, 395 (1988).

[19] M.S. Morris, K.S. Thorne and U. Yurtsever, Phys. Rev. Lett, 61, 1446 (1988).

[20] M. Visser, Scale anomalies imply violation of the averaged null energy condition, Phys. Lett. B349, 443 (1995); gr-qc/9409043

[21] M. Visser, Gravitational vacuum polarization I: Energy conditions in the Hartle-Hawking vacuum, Physical Review D54 (1996) 5103-5115; grqc/9604007.

[22] M. Visser, Gravitational vacuum polarization II: Energy conditions in the Boulware vacuum, Physical Review D54 (1996) 5116-5122; gr-qc/9604008.

[23] M. Visser, Gravitational vacuum polarization III: Energy conditions in the (1+1) Schwarzschild spacetime, Physical Review D54 (1996) 5123-5128; gr-qc/9604009.

[24] M. Visser, Gravitational vacuum polarization IV: Energy conditions in the Unruh vacuum, Physical Review D15 in press (15 July 1997); grqc/9703001. 
[25] J. Wambsganss, R. Chen. G. Xu, and J.P. Ostriker, Effects of weak gravitational lensing from large-scale structure on the determination of $q_{0}$, Astrophysical Journal, 475 (1997) L81-L84.

[26] V. Sanhi, H. Feldman, and A. Stebbins, Loitering Universe, Astrophysical Journal, 385 (1992) 1.

[27] H. Feldman and A.E. Evrard, Structure in a loitering universe, astroph/9212002; International Journal of Modern Physics D2 (1993) 113-122.

[28] M. Kamionkowski and N. Toumbas, A low density closed universe, Physical Review Letters 77 (1996) 587.

[29] L.F. Bloomfield Torres and I. Waga, Decaying $\Lambda$ cosmologies and statistical properties of gravitational lenses, astro-ph/9504101.

[30] V. Silveira and I. Waga, Cosmological properties of a class of $\Lambda$ decaying cosmologies, astro-ph/9703185.

[31] Particle Data Group, Review of Particle Properties, Phys. Rev. D 54 (1996) $1-720$.

[32] E. W. Kolb and M. S. Turner, The Early Universe, (Addison-Wesley, Redwood City, 1990).

[33] A. D. Linde, Inflation and Quantum Cosmology, (Academic, Boston, 1990).

[34] I. Moss, Quantum Theory, Black Holes, and Inflation, (Wiley, Chichester, 1996).

[35] W. H. Press, B. S. Ryden, and D. N. Spergel, Single mechanism for generating large scale structure and providing dark missing matter, Physical Review Letters 64 (1990) 1084. 